\title{
Aflatoxin exposure and micronutrient deficiency among young children from Guinea
}

\author{
S. Watson, G. Chen and Y.Y. Gong \\ Institute for Global Food Security, School of Biological Sciences, Queen's University Belfast, Belfast BT9 5BN, UK
}

The causes of high prevalence of micronutrient deficiency in children from developing countries are multifactorial and not simply a direct result of inadequate dietary intake (1). It has been postulated that aflatoxin, a type of mycotoxin, that frequently affects dietary staples such as groundnuts and maize, may impair gut permeability, reduce nutrient bioavailability, and consequently exacerbate micronutrient deficiency (2). Most of the evidence supporting this relationship is from animal feeding studies (3) and the limited evidence in human subjects is inconsistent. The aim of this study was to examine the relationship between aflatoxin exposure and micronutrient deficiency among young children from Guinea.

A total of 305 children (50.5\% male; mean age $28.8 \pm 8.4$ months) were randomly recruited at harvest (October) from subsistence farming households located within the region of Kindia, West Guinea, an area where groundnuts are a dietary staple and aflatoxin exposure is high. To assess seasonal variations, six months post-harvest, the children were followed up again where possible $(\mathrm{n}=288)$. Aflatoxin exposure was assessed in blood samples using the aflatoxin-albumin adduct (AF-alb) biomarker measured by an ELISA method. Plasma micronutrient concentrations including vitamin A, vitamin E, beta-carotene and zinc were measured using HPLC methods. Socio-demographic and dietary information were recorded.

At harvest $88.2 \%$ and at post-harvest $93.4 \%$ of blood samples had detectable AF-alb concentrations. The geometric mean AF-alb concentration at harvest was significantly lower than at post-harvest (12.70, $95 \% \mathrm{CI}$ : 10.91, $14 \cdot 73$ vs. $16 \cdot 29,95 \% \mathrm{CI}: 14 \cdot 44,18 \cdot 54$ $\mathrm{pg} / \mathrm{mg} ; P=0.009)$. Vitamin A, vitamin E and beta-carotene median concentrations increased significantly from harvest to post-harvest $(P<0.001)$; whereas zinc concentrations decreased $(P=0.008)$. Similarly, more children were deficient in vitamin A $(78.9 \%$ vs. $60.5 \%, P<0.001)$ and vitamin E $(44.7 \%$ vs. $26.3 \%, P=0.001)$ at harvest than at post-harvest; while more children were deficient in zinc at post-harvest than at harvest $(75.2 \%$ vs. $57.5 \%, P=0.001)$. Logistic regression was undertaken to determine the association between micronutrient deficiency and aflatoxin exposure at each time point. As shown in the table below, children in the highest aflatoxin exposure group (Q4), compared to the lowest (Q1) were 1.98 (95\% CI: 1.00, 3.92) and 3.56 (95\% CI: 1.13, 11.15) times more likely to be zinc and vitamin A deficient at harvest. Also, children in the second lowest aflatoxin exposure group (Q2) were 2.05 (95\% CI: 1.03, 4.07) times more likely to be zinc deficient compared to the lowest exposed group (Q1). No correlation was found at post-harvest.

\begin{tabular}{|c|c|c|c|c|}
\hline Micronutrients & \multicolumn{4}{|c|}{ Odds Ratio $(95 \% \mathrm{CI})$} \\
\hline Zinc & Ref & $2.05(1.03,4.07)^{*}$ & $1.74(0.89,3.40)$ & $1.98(1.00,3.92)^{*}$ \\
\hline Vitamin A & Ref & $0.91(0.35,2.41)$ & $1.57(0.60,4.11)$ & $3.56(1.13,11.15)^{*}$ \\
\hline Beta Carotene & Ref & $0.67(0.30,1.49)$ & $0.99(0.45,2.19)$ & $1.28(0.59,2.80)$ \\
\hline
\end{tabular}

Analysed using logistic regression: $* \mathrm{P} \leqslant 0 \cdot 05, * * \mathrm{P} \leqslant 0 \cdot 01, * * * \mathrm{P} \leqslant 0 \cdot 001$. Data presented as odd ratios (95\% CI). Adjusted for age, gender, socio-economic status and breastfeeding status. Q is quartile. Q1 is the reference group (lowest exposed) and Q4 is the highest exposed group.

Aflatoxin exposure and micronutrient deficiencies were highly prevalent in this population group and were influenced by season. Children with high aflatoxin exposure levels were more likely to be zinc and vitamin A deficient at harvest. Targeting aflatoxin exposure may help the outcomes of micronutrient supplementation trials.

1. Ruel MT \& Alderman H (2013) Lancet 382, 536-531.

2. Gong YY, Turner PC, Hall AJ et al. (2008) In: Mycotoxins: Detection Methods, Management, Public Health, and Agricultural Trade, pp. 53-66. Wallingford, UK.

3. Williams JH, Phillips TD, Jolly PE et al. (2004) Am J Clin Nutr 80, 1106-1122. 